\title{
Evaluation of Cucumber Hybrids for Yield and Quality Under Plastic Houseand Open Field Conditions
}

\author{
Ishwori Prasad Gautam ${ }^{1 *}$, Navin Gopal Pradhan', Sujan Subedi ${ }^{1}$ and Manis Kumar Thakur ${ }^{2}$ \\ ${ }^{\prime}$ National Horticulture Research Centre, Province 3, Khumaltar, Lalitpur, \\ ${ }^{2}$ Directorate of Agriculture Research, Province 1, Tarahara, Sunsari* \\ "Corresponding Author's Email: ishworigautam@gmail.com \\ "Orcid ID: https://Orcid.org/0000-0003-0682-9306
}

Received on: 24 September 2020, Revised on:16 January 2021, Accepted on: 3 March 2021

\begin{abstract}
Low yield of off-season cucumber due to use of open-pollinated varieties with high male to female ration, long gestation period and high incidence of diseases are the major problems of cucumber production in Nepal. The use of imported hybrids varieties during the off-season and plastic film technology has shown to lead to enhanced growth and higher yield in recent years. However, there is a little work on hybrid cucumber research and their testing in Nepal. Therefore, experiments were conducted atthe National Horticulture Research Center (NHRC), Directorate of Agricultural Research (DoAR), Tarahara and Farmer's field in Dhankuta district accordingly to select best hybrids. Sixteen genotypes including best Indian hybrid Dynasty and 3 open-pollinated check genotypes were evaluated at NHRC, Khumaltar during the years 2018 and 2019 in Randomized Complete Block Design (RCBD) with three replications under the plastic house. Previously selected seven genotypes were evaluated in DoAR, Tarahara in 3 replications and best hybrid was compared with farmers' varieties in Dhankuta at open field conditions. Tested genotypes showed a significant difference in yield and other parameters like male to female ratio, early flowering, vine length, disease scoring etc. both under the plastic house and open field conditions. The new hybrids HRD CUM 009 x $003(107.05 \mathrm{mt} / \mathrm{ha})$, HRD CUM $006 \mathrm{x}$ $003(91.55 \mathrm{mt} / \mathrm{ha})$ and HRD CUM $004 \times 001(88.10 \mathrm{mt} / \mathrm{ha})$ were promising due to higher yield, earlier to $50 \%$ female and male flowering, a greater number of female flowers, short vine length, higher number of fruits per plants and earliness harvesting than other cultivated varieties. Most of the tested hybrids produced more than double yield than open-pollinated genotypes.
\end{abstract}

Keywords: Cucumber, hybrids development, plastic house, yield potentiality

\section{Introduction:}

Cucumber (Cucumis sativus L., $2 \mathrm{n}=2 \mathrm{x}=14$ ) is the member of the economically important family of cucurbitaceae. It is warm-season crops mainly grown in the tropical and sub-tropical region of the world. It is very susceptible to frost. The temperature ranges 18 $24^{\circ} \mathrm{C}$ is optimum for cucumber production. In Nepal, it is grown commercially during the summer season.
Cucumber is used asa Salad dish, Sandwich, Rayta and preparation of Pickles and Pizza etc.It is also useful for people suffering from kidney problems, constipation, jaundice and indigestion (Pal et al. 2017). Oil extraction from seeds is considered good for the brain and body. In Nepal, it is the seventh important vegetable crop in terms of area. The area, production and productivity of 
cucumber in Nepal is 10812 ha, $172566 \mathrm{mt}$ and 15.96 $\mathrm{mt} /$ ha respectively (MoALD, 2019).

There is an increasing demand of cucumbers during the off-season due to its multiple uses. Presently, it becomesthemost preferred and cash-generating vegetables throughout the year. Some commercial farmers near the roads and markets started for the growing of this crop during the off-season to get remunerative price. However, there is lack of climate resilience and short duration and high yielding varieties and technology for year-round production of cucumber. The productivity of cucumber is very low in Nepal (15.96mt/ha) (MoALD,2019) compared to Asia (49.61 $\mathrm{mt} / \mathrm{ha}$ ) and world average productivity $(39.34 \mathrm{mt} / \mathrm{ha})$ (FAO Stat, 2021). It could be due to the use of local and open-pollinated varieties growing inthe open field. Most of the farmers used their local varieties for normal season production in the open field. Out of 38 registered and recommended varieties, the seed production is possible for only two open-pollinated varieties Kusle and Bhaktapur Local ted (OP) varieties in Nepal due to lack of our hybrid and hybrid seed production technologies. Among these OP varieties, the Kusle variety is not preferred by farmers due to severely affected by the virus and powdery mildew and is in the nearly de-notified stage. The next, OP variety Bhaktpur local is popular among the farmersfor main season production. however, it has many drawbacks; as it takesa long time for fruiting, short productive life, high male to female ratio,high number of misshapen fruits, presence of a cavity in the centre and susceptibility topowdery and downy mildew and viruses (Gautam et al. 2008). Therefore, most of the farmers use hybrid varieties developed by other countries for higher and early production during different seasons.

Most of the farmers started growing hybrids cucumber under the plastic house in accessible areas during the off-season for fetching remunerative returns due to their adaptability and high yield under plastic house condition. Compared to open field very high yield has been reported even under naturally ventilated poly houses (Srivastav and Singh, 1997 and Gautam et.al. 2008). Itis reported that plastic film technology enable scropstomature 5 to 20 days earlier than normal, increases the yield by 30 to $50 \%$ and increases output value by 40 to $50 \%$ and prolongs harvesting period of vegetables by 40 to 60 days (Rongsen,1994). The large quantity of hybrid seeds of cucumber is imported from aboard to fulfil the high seed demand. It is reported that cucumber growers used only $19 \%$ of open-pollinated seeds and 78\% of hybrids seeds were imported from aboard (Kafle and Joshi, 2018). Hybrids varietiesare popular due to early maturity, low male to female ratio, short plant height, wide adaptability and no specific to climate and season. Considering these problems and opportunities, National Horticulture Research Center has started to develop own cucumber hybrid by introducing some exotic gynoceious lines and crossing them with our local genetic materials in the past and become successes to produce the different hybrid.Hence, the present study was conducted to verify the past results and find out the earlyand high yielding cucumber hybrids under the plastic house and open field conditions.

\section{Materials and Methods}

The experiment was conducted under the plastic house to find out the quality and yield potential of new cucumber hybrids developed by NHRC during the years 2018 and 2019. Best six hybrids selected from NHRC were tested simultaneously in the open field as CVT at DoAR Tarahara and one best hybrid selected for the eastern region was tested at farmers' fields in the open field as FFT in Dhankuta district.

Sixteen cucumber genotypes (12 NHRC hybrids, 3 open-pollinated and one most popular Indian hybrid dynasty) were selected for experimentation under the plastic house at NHRC, Khumaltar.The experiment was laid out in a randomized block design with three replications. The seed was sown on $15^{\text {th }}$ Feb 2018

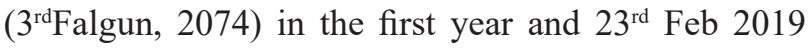
(11 ${ }^{\text {th }}$ Falgun) in the second year in polythene bag for healthyand uniform seedlings. Twenty-eight- and thirtydaysold seedlings were transplanted under the plastic house in first and second years respectively. Seedlings were transplanted in square plot adjusted with 4 plants at a spacing of $1 \mathrm{~m} \times 1 \mathrm{~m}$ at row to row and plant to plant distance.Manures and fertilizers were applied at the rate of $3 \mathrm{~kg}$ FYM, $50 \mathrm{~g}$ mustard oil cake, $50 \mathrm{~g}$ bone meal, $20 \mathrm{~g}$ DAP, 20g MOP and $3 \mathrm{~g}$ each micronutrient (Bio zyme, 
Zinc, and Borax) in each pit before planting in the first year. In the second year $3 \mathrm{~kg}$ FYM, 100g mustard oil cake, 100g bone meal, 20g DAP, 20g MOP and $3 \mathrm{~g}$ each micronutrient (Bio zyme, Zinc, and Borax) were applied before planting in each pit. A total of $30 \mathrm{~g}$ urea was top-dressed equally in 3 split doses at 20, 35 and 50 days after transplanting in both years. Along with the urea 3g,each micronutrient (Bio zyme, Zinc, and Borax) were applied in the ring at the second top-dressing time. Other insecticides, fungicide and Vircon-H (antivirus) were applied when needed. Three shoots per plant were maintained in a single plant by removing other shoots by secateurs. The plants were trained by the help of bamboo, GI wire and plastic rope. Other management practices were carried out as per the package of practices recommended by NHRC. Observations were recorded on days to first flowering, female flowering node, male to female flower ratio, days to the first harvest, individual fruit weight $(\mathrm{g})$, fruit girth $(\mathrm{mm})$ and length $(\mathrm{cm})$, marketable, un-marketable yieldtons per hectare. Observations on quality parameters like fruit size, appearance, taste, hollowness and hedonic rating were also recordedin different scales (Table 6) by the panel of scientists.

\section{Coordinated Varietal Trial of Cucumber Hybrids at DoAR, Tarahara}

A total of seven cucumber genotypes including two open-pollinated and 5 hybrids selected from previous years were tested at DoAR, Tarahara to find out the best varieties at eastern Terai conditions. The experiment was laid out in a RCBD with 3 replications. Cucumber seeds were sown on $28^{\text {th }}$ February in poly-pots and 35 days old seedlings were planted in the open field on $2^{\text {nd }}$ May 2018. The individual plot size for the experiment was $9.6 \mathrm{~m}^{2}(4.8 \mathrm{~m} \mathrm{x} 2.0 \mathrm{~m})$ with one bed per plot at a distance of $2 \mathrm{~m} \times 1 \mathrm{~m}$ row to row and plant to plant distance. Compost and fertilizers were applied at the rate of $5 \mathrm{~kg}$ compost, $20 \mathrm{~g}$ DAP, $20 \mathrm{~g}$ potash and $3 \mathrm{~g}$ borax per plant at the time of transplanting. Urea was top-dressed at the rate of $20 \mathrm{~g} /$ plant equally at two split doses;at 25 and 50 days after transplanting. Data were recorded on days to $50 \%$ male and female flowering, firstfemale flowering node, branch numbers, days to first harvesting, plant height, length and diameter of fruits, number of fruits/plants, marketable and un- marketable fruits, total yield, insect and disease scoring and farmers response.

\section{Farmers Field Trial of Cucumber at NACRE, Dhankuta}

One selected cucumber hybrid HRD CUM 009x HRD CUM 003 and one locally adopted variety HRDCUC003 (Dhankuta Local) were tested as FFT inNCRP, Dhankuta farm and farmers' fields in Dhankuta district. Seedlings were sown in poly-pots on $15^{\text {th }}$ April2018 and 25 days old seedlings were transplanted on $10^{\text {th }}$ May 2018 in open field conditions. Five seedlings of both genotypes were planted at a spacing of $1.2 \mathrm{~m} \mathrm{x}$ $1.2 \mathrm{~m}$ in four replications; as one replication in NCRP farm and three in 3 farmers' fields.Manure and fertilizer were applied at the rate of $4.5 \mathrm{~kg}$ compost, $12.5 \mathrm{~g}$ DAP, $20 \mathrm{~g}$ urea and $24 \mathrm{~g}$ potash per plant. Observations were recorded on days to first harvest, length and diameter of fruits, vine length and farmers response. The harvesting was completed on $3^{\text {rd }}$ week of August 2018.

\section{Results}

Number of first female flowering node and male to female flower ratio

Theeffectof genotypesonthe first female flowering node and male to female ratio showed a significant difference in both years and their pooled analysis. The pooled data showed that the hybrid genotypes HRDCUC-009 x 003 and HRDCUC-006 x 008 produced the female flower at the lowest node (6.0) followed by HRDCUC-009 x 001 (6.5) and HRDCUC-004 x 003 (7.0).The open genotypes and HRDCUC-004 x 001produced the first female flower in the highest node (27.0) followed by Bhaktpur Local (15.5 nodes). The detail record of each year of different genotypes is presented in table 1 .

The pooled analysis showed the significantly the lowest male to female flower ratio (1:02) was recorded in new hybrids HRDCUC-006 x 001 and HRDCUC-004 x 003 and they were statistically at par with HRDCUC-009 $\mathrm{x}$ 008, HRDCUC-007 x 001 (1: 07) and HRDCUC-006 x 007 and HRDCUC-006 x 008 (1:04). The open pollinated variety Bhaktapur local produced the highest male to female ratio (8.6:1) followed by HRD CUM $004 \times 001(2.5: 1)$ The detail observations of each genotype in each year are given in table 1. 
Table 1.Number of first female flowering and male flowering node of cucumber under the plastic house at NHRC, Khumaltar during 2018 and 2019

\begin{tabular}{|c|c|c|c|c|c|c|}
\hline Genotypes & Firs & e flowe & lode & Male & male flowe & ratio \\
\hline Year & 2018 & 2019 & Pooled & 2018 & 2019 & Pooled \\
\hline HRD CUM 001 & $16 b$ & $15 b$ & $15.5 b$ & $9.2: 1 f$ & $8.1: 1 f$ & $8.6: 1 f$ \\
\hline HRD CUM 004 x 001 & $28 a$ & $26 a$ & $27.0 a$ & $2.7: 1 e$ & $2.3: 1 e$ & $2.5: 1 e$ \\
\hline HRD CUM $009 \times 003$ & $8 \mathrm{efg}$ & $6 g$ & $6.0 h$ & $1: 3.2 c$ & $1: 2.6 c$ & $1: 2.9 c d$ \\
\hline HRD CUM 009 x 007 & $10 c d e$ & $10 \mathrm{de}$ & 10.0 de & $1: 5.1 d$ & $1: 4.4 d$ & $1: 4.7 e$ \\
\hline HRD CUM 006 x 003 & $8 \mathrm{efg}$ & 8 efg & $8.0 \mathrm{efgh}$ & $1: 0.3 a$ & $1: 0.4 a$ & $1: 0.3 a$ \\
\hline Dynasty & $10 c d e$ & $10 d e$ & $10.0 \mathrm{de}$ & $1: 0.6 a$ & $1: 0.6 a$ & $1: 0.6 a b$ \\
\hline HRD CUM 009 x 001 & $6 g$ & $7 f g$ & $6.5 g h$ & $1: 3.3 c$ & $1: 2.2 b c$ & $1: 2.7 c d$ \\
\hline HRD CUM 009 x 008 & $9 d e f$ & $9 e f$ & $9.0 e f$ & 1:0.1 $a$ & 1:0.4 a & $1: 0.3 a$ \\
\hline HRD CUM 006 x 001 & $8 \mathrm{efg}$ & $8 \mathrm{efg}$ & 8.0 efgh & 1: $0.3 a$ & $1: 0.2 a$ & $1: 0.2 a$ \\
\hline HRD CUM 006 x 007 & $8 \mathrm{efg}$ & $8 \mathrm{efg}$ & 8.0 efgh & 1: $0.4 a$ & $1: 0.4 a$ & $1: 0.4 a$ \\
\hline HRD CUM 006 x 008 & $6 g$ & $6 g$ & $6.0 h$ & $1: 0.4 a$ & $1: 0.4 a$ & $1: 0.4 a$ \\
\hline HRD CUM 004 x 003 & $7 f g$ & $7 f g$ & $7.0 \mathrm{fgh}$ & $1: 0.2 a$ & $1: 0.3 a$ & $1: 0.2 a$ \\
\hline HRD CUM 004 x 007 & $12 c$ & $12 c d$ & $12.0 c d$ & $1: 0.3 a$ & $1: 0.4 a$ & $1: 0.3 a$ \\
\hline HRD CUM 004 x 008 & $8 \mathrm{efg}$ & $9 c f$ & $8.5 \mathrm{efg}$ & $1: 2.1 \mathrm{~b}$ & $1: 1.3 a b$ & $1: 1.7 b c$ \\
\hline HRD CUM 007 & $11 \mathrm{~cd}$ & $13 b c$ & $12.5 c$ & $1: 1.9 \mathrm{~b}$ & $1: 1.9 b c$ & $1: 1.9 c$ \\
\hline HRD CUM 008 & $12 c$ & $13 b c$ & $12.5 c$ & $1: 3.9 c$ & $1: 3.8 d$ & $1: 3.8 \mathrm{de}$ \\
\hline F-test & $* * *$ & $* * *$ & $* * *$ & $* * *$ & $* * *$ & $* * *$ \\
\hline LSD & 2.102 & 2.639 & 2.223 & 0.951 & 1.095 & 1.110 \\
\hline $\mathrm{CV}$ & 9.2 & 11.4 & 9.9 & 15 & 17.1 & 25.2 \\
\hline
\end{tabular}

\section{Daysto first harvesting}

The first and second-year data and their pooled analysis showed a highly significant difference on days to first harvesting among the tested genotypes. The Indian hybrid dynasty was harvested earlier (49) days after transplanting followed by HRD CUM $006 \times 001$ (57.0 days) followed by HRD CUM 009 x 001 and HRD CUM 009 x 008 (59.0) days after transplanting. The detail first harvesting days of each genotype in each year is presented in table 2 .

\section{Weight of individual fruit}

The genotypes showed highly significant and significant results on individual fruit weight in first and second years and pooled analysis respectively. The maximum fruit weight $(656 \mathrm{~g})$ was recorded on hybrid genotype HRD CUM $009 \times 003$ and it was at statistically par with HRD CUM 006 x 003 (568 g), HRD CUM 008 (553g) and HRD CUM $009 \times 007$ (535g), whereas the minimum individual fruit weight (396g) was observed in Indian hybrid Dynasty followed by HRD CUM 009 x 001 (440g), HRD CUM 006 x 001(445g), HRD CUM 006 x 007 (454g), HRD CUM 009 x 008 (474g), HRD CUM 006 x 008(496g) and HRD CUM 009 x $008(496 \mathrm{~g})$. For fresh markets, most of the consumers preferred small fruit size due to its good taste and absence of hollowness. The detail observation of each genotype in a different year and their pooled analysis is presented in table 2 .

Table 2. Average days to first harvest and weight of individual cucumber fruit under the plastic house atNHRC, Khumaltar during 2018 and 2019

\begin{tabular}{|l|c|c|c|c|c|c|}
\hline \multicolumn{1}{|c|}{ Genotypes } & \multicolumn{3}{c|}{ Days to first harvesting } & \multicolumn{3}{c|}{ Weight of individual fruit (g) } \\
\hline Years & $\mathbf{2 0 1 8}$ & $\mathbf{2 0 1 9}$ & Pooled & $\mathbf{2 0 1 8}$ & $\mathbf{2 0 1 9}$ & Pooled \\
\hline HRD CUM 001 & $69 b$ & $54 a b$ & $61.5 a b$ & $639 a$ & $393 a$ & $516 a b c$ \\
\hline
\end{tabular}




\begin{tabular}{|c|c|c|c|c|c|c|}
\hline Genotypes & \multicolumn{3}{|c|}{ Days to first harvesting } & \multicolumn{3}{|c|}{ Weight of individual fruit (g) } \\
\hline HRD CUM 004 x 001 & $64 b c d$ & $50 b c$ & $57.0 a b$ & 502 bcde & $629 \mathrm{bcd}$ & $566 b c$ \\
\hline HRD CUM $009 \times 003$ & $62 c d$ & $51 b c$ & $56.5 a b$ & 596 ef & $716 d$ & $656 c$ \\
\hline HRD CUM $009 \times 007$ & $61 d$ & $50 b c$ & $55.5 a b$ & $419 a b c$ & $651 c d$ & $535 a b c$ \\
\hline HRD CUM $006 \times 003$ & $60 \mathrm{~cd}$ & $52 b c$ & $56.0 a b$ & 569 def & $567 \mathrm{abcd}$ & $568 b c$ \\
\hline Dynasty & $49 e$ & $45 c$ & $47.0 \mathrm{~b}$ & $342 a$ & $451 a b$ & $396 a$ \\
\hline HRD CUM 009 x 001 & $59 d$ & $51 b c$ & $55.0 a b$ & $382 a b$ & $497 a b c$ & $440 a b$ \\
\hline HRD CUM $009 \times 008$ & $59 d$ & $55 a b$ & $57.0 a b$ & $429 a b c$ & $519 a b c$ & $474 a b$ \\
\hline HRD CUM $006 \times 001$ & $57 d$ & $50 b c$ & $53.5 a b$ & $398 a b c$ & $492 a b c$ & $445 a b$ \\
\hline HRD CUM 006 x 007 & $62 c d$ & $56 a b$ & $59.0 a b$ & $427 a b c$ & $481 a b c$ & $454 a b$ \\
\hline HRD CUM 006 x 008 & $61 c d$ & $49 b c$ & $55.0 a b$ & 484 bcde & $508 a b c$ & $496 a b$ \\
\hline HRD CUM $004 \times 003$ & $62 c d$ & $53 \mathrm{bcd}$ & $57.5 a b$ & 454 abcd & $600 \mathrm{bcd}$ & $527 a b c$ \\
\hline HRD CUM 004 x 007 & $62 c d$ & $51 a b$ & $56.5 a b$ & $523 c d e f$ & $485 a b c$ & $504 a b$ \\
\hline HRD CUM $004 \times 008$ & $59 c d$ & $51 b c$ & $55.0 a b$ & $417 a b c$ & $642 \mathrm{bcd}$ & $530 a b c$ \\
\hline HRD CUM 007 & $78 a$ & $56 a b$ & $67.0 a b$ & 455 abcd & $613 \mathrm{bcd}$ & $534 a b c$ \\
\hline HRD CUM 008 & $67 b c$ & $60 a$ & $63.5 a b$ & 475 abcde & $631 \mathrm{bcd}$ & $553 b c$ \\
\hline F- test & $* * *$ & $* * *$ & $* *$ & $* * *$ & $*$ & $*$ \\
\hline LSD & 6.387 & 6.462 & 13.97 & 117.6 & 164.1 & 124.9 \\
\hline $\mathrm{CV} \%$ & 4.7 & 5.7 & 11.4 & 15.0 & 17.7 & 21.2 \\
\hline
\end{tabular}

\section{Length and girth of fruits}

The length and girth of fruits of different genotypes showed highly significant and significant results respectively in each year and pooled analysis (Table 3). The pooled data showed the maximum fruit length $(30.45 \mathrm{~cm})$ on hybrid genotype HRD CUM 006 x 003 followed by HRD CUM $009 \times 003(29.91 \mathrm{~cm})$ and HRD CUM $004 \times 003(29.10 \mathrm{~cm})$, whereas it was recorded the minimum $(24.34 \mathrm{~cm})$ on HRD CUM $006 \times 007$. However, there was some variation on fruit length on the first and second years. Similarly, the maximum fruit girth $(63.79 \mathrm{~mm})$ was recorded on open-pollinated genotype HRD CUM 007 of both years combined data and it was statistically at par with OP genotype HRD CUM $008(63.72 \mathrm{~mm})$ and the minimum $(54.32 \mathrm{~mm})$ on Indian hybrid Dynasty. Other genotypes showed the values within the minimum and maximum range. The detailed analysis of fruit girth in each year is presentedin table 3 .

Table 3. Average length and girth of cucumber fruit under the plastic house at NHRC, Khumaltar during 2018 and 2019

\begin{tabular}{|c|c|c|c|c|c|c|}
\hline \multirow{2}{*}{$\begin{array}{c}\text { Genotypes } \\
\text { Years }\end{array}$} & \multicolumn{3}{|c|}{ Fruit Length (cm) } & \multicolumn{3}{|c|}{ Fruit girth (mm) } \\
\hline & 2018 & 2019 & Pooled & 2018 & 2019 & Pooled \\
\hline HRD CUM 001 & $28.53 \mathrm{de}$ & $21.7 a$ & $25.12 a$ & $66.73 \mathrm{~d}$ & $59.62 \mathrm{a}$ & $63.18 \mathrm{bc}$ \\
\hline HRD CUM 004 x 001 & $23.73 a b c$ & $24.72 b c$ & $26.23 a$ & $60.23 \mathrm{bc}$ & $63.83 \mathrm{abcd}$ & $62.03 \mathrm{bc}$ \\
\hline HRD CUM 009 x 003 & $28.33 d e$ & $31.49 \mathrm{~g}$ & $29.91 b c$ & $59.23 \mathrm{bc}$ & $60.38 \mathrm{a}$ & $59.81 \mathrm{bc}$ \\
\hline HRD CUM 009 × 007 & $23.8 a b c$ & $26.11 b c d$ & $24.96 a$ & $56.7 \mathrm{bc}$ & $62.95 \mathrm{abcd}$ & $59.83 \mathrm{bc}$ \\
\hline HRD CUM 006 x 003 & $30.7 e$ & $30.19 \mathrm{fg}$ & $30.45 c$ & $60.57 \mathrm{c}$ & $59.03 \mathrm{a}$ & $59.80 \mathrm{bc}$ \\
\hline Dynasty & $21.07 a$ & $28 \mathrm{def}$ & $24.54 a$ & $48.98 \mathrm{a}$ & $59.66 \mathrm{a}$ & $54.32 \mathrm{a}$ \\
\hline HRD CUM 009 x 001 & $25.7 b c d$ & $23.43 a b$ & $24.57 a$ & $54.79 \mathrm{~b}$ & $61.12 \mathrm{abc}$ & $57.96 \mathrm{ab}$ \\
\hline HRD CUM $009 \times 008$ & $24.03 a b c$ & $25.65 \mathrm{bcd}$ & $24.84 a$ & $58.67 \mathrm{bc}$ & $60.74 \mathrm{a}$ & $59.71 \mathrm{bc}$ \\
\hline HRD CUM 006 x 001 & $24.47 a b c d$ & $25.09 b c$ & $24.78 a$ & $55.1 \mathrm{~b}$ & $61.09 \mathrm{ab}$ & $58.10 \mathrm{ab}$ \\
\hline HRD CUM 006 × 007 & $23.7 a b c$ & $24.97 b c$ & $24.34 a$ & $57.57 \mathrm{bc}$ & $61.62 \mathrm{abcd}$ & $59.60 \mathrm{bc}$ \\
\hline HRD CUM 006 × 008 & 27.2 cde & $24.5 b c$ & $25.85 a$ & $57.2 \mathrm{bc}$ & $60.09 \mathrm{a}$ & $58.65 \mathrm{abc}$ \\
\hline
\end{tabular}




\begin{tabular}{|l|c|c|c|c|c|c|}
\hline \multicolumn{1}{|c|}{ Genotypes } & \multicolumn{3}{c|}{ Fruit Length (cm) } & \multicolumn{3}{c|}{ Fruit girth (mm) } \\
\hline \multicolumn{1}{|c|}{ Years } & $\mathbf{2 0 1 8}$ & $\mathbf{2 0 1 9}$ & Pooled & $\mathbf{2 0 1 8}$ & $\mathbf{2 0 1 9}$ & Pooled \\
\hline HRD CUM 004 x 003 & $28.68 \mathrm{de}$ & $29.51 \mathrm{efg}$ & $29.10 \mathrm{bc}$ & $59.1 \mathrm{bc}$ & $59.06 \mathrm{a}$ & $59.08 \mathrm{abc}$ \\
\hline HRD CUM 004 x 007 & $28.57 \mathrm{de}$ & $23.68 \mathrm{ab}$ & $26.13 \mathrm{a}$ & $57.47 \mathrm{bc}$ & $60.37 \mathrm{a}$ & $58.92 \mathrm{abc}$ \\
\hline HRD CUM 004 x 008 & $25.47 \mathrm{bcd}$ & $29.33 \mathrm{efg}$ & $27.40 \mathrm{abc}$ & $55.6 \mathrm{bc}$ & $63.61 \mathrm{abcd}$ & $59.61 \mathrm{bc}$ \\
\hline HRD CUM 007 & $23.2 \mathrm{abc}$ & $27.09 \mathrm{cde}$ & $25.15 \mathrm{a}$ & $60.97 \mathrm{c}$ & $66.61 \mathrm{bcd}$ & $63.79 \mathrm{c}$ \\
\hline HRD CUM 008 & $22.7 \mathrm{ab}$ & $20.19 \mathrm{bcd}$ & $21.45 \mathrm{a}$ & $60.87 \mathrm{c}$ & $66.56 \mathrm{bd}$ & $63.72 \mathrm{c}$ \\
\hline F- test & $* * *$ & $* * *$ & $* * *$ & $* * *$ & $*$ & $*$ \\
\hline LSD & 3.647 & 2.499 & 3.062 & 4.559 & 4.820 & 4.540 \\
\hline CV\% & 8.6 & 5.7 & 10.3 & 4.7 & 4.7 & 6.5 \\
\hline
\end{tabular}

\section{Number of fruits}

The average number of fruits per plants is directly related to the total yield of the crop. In this experiment, the tested genotypes showed a highly significant difference in the number of fruits in both years and their pooled analysis. The number of fruits/plants varied from 9 22 in the first year, 6-37 in the second year and 8-27 in pooled data. The pooled data showed the maximum numbers fruits (27) on hybrid genotype HRD CUM
$006 \times 008$ and the minimum (8) was observed on op genotype HRD CUM 001 and the minimum data was statistically at par with Indian hybrid Dynasty (10). The detail observation in each year of each genotype is presented in table 4.The low number of fruits in Indian hybrid was due to early fruiting. small plant height, small size of fruits and short crop duration.

Table 4.Average numbers of fruits per plant of cucumber under the plastic house at NHRC, Khumaltar during 2018 and 2019

\begin{tabular}{|c|c|c|c|}
\hline Genotypes & \multicolumn{3}{|c|}{ Fruit numbers/plant } \\
\hline Years & 2018 & 2019 & Pooled \\
\hline HRD CUM 001 & $9 \mathrm{ab}$ & $6 a$ & $8 a$ \\
\hline HRD CUM 004 x 001 & 14 abcdef & 26 cde & $20 b c$ \\
\hline HRD CUM 009 x 003 & 17 bcdef & $24 c d$ & $21 b c$ \\
\hline HRD CUM 009 x 007 & 13 abcde & $30 \mathrm{de}$ & $22 b c$ \\
\hline HRD CUM 006 x 003 & 18 cdef & 26 cde & $22 b c$ \\
\hline Dynasty & $9 \mathrm{a}$ & $10 a b$ & $10 a$ \\
\hline HRD CUM 009 x 001 & 20 cdef & $20 \mathrm{bcd}$ & $20 b c$ \\
\hline HRD CUM 009 × 008 & $21 \mathrm{def}$ & $23 c d$ & $22 b c$ \\
\hline HRD CUM 006 x 001 & $21 \mathrm{ef}$ & $11 a b$ & $16 a b$ \\
\hline HRD CUM 006 x 007 & $22 \mathrm{f}$ & $19 b c d$ & $21 b c$ \\
\hline HRD CUM 006 x 008 & $17 \mathrm{cdef}$ & $37 f$ & $27 c$ \\
\hline HRD CUM 004 x 003 & 20 cdef & $28 c d e$ & $24 b c$ \\
\hline HRD CUM 004 x 007 & 14 abcdef & $17 a b c$ & $16 a b$ \\
\hline HRD CUM 004 x 008 & 17 bcdef & $24 c d$ & $21 b c$ \\
\hline HRD CUM 007 & $12 a b c$ & $23 c d$ & $18 b c$ \\
\hline HRD CUM 008 & $12 \mathrm{abc}$ & $16 a b c$ & $14 a b$ \\
\hline F-value & $* *$ & $* * *$ & $* * *$ \\
\hline LSD & 7.469 & 10.802 & 8.356 \\
\hline CV\% & 28.3 & 30.5 & 39.2 \\
\hline
\end{tabular}




\section{Yield Per Plant and Total Yield (mt/ha)}

The genotypes differed significantly on cucumber fruit yield/plant and total yield ( $\mathrm{t} / \mathrm{ha}$ ) in both years and pooled analysis. The yield/plant was recorded the highest $(13.38 \mathrm{~kg} / \mathrm{plant})$ on cucumber hybrid HRD CUM 009 x 003 followed by HRD CUM 006 x 008 $(12.78 \mathrm{~kg} / \mathrm{plant})$ and HRD CUM 004 x $003(12.50 \mathrm{~kg} /$ plant) in the combinedanalysis. The lowest mean yield $(3.26 \mathrm{~kg} /$ plant was observed on Indian hybrid Dynasty followed by Bhaktpur local, i.e. HRDCUC 001 (3.83 $\mathrm{kg} /$ plant). The variation on yield per plant is presented in table 5 .

Similarly, genotypes showed the highly significant difference on adjusted yield per hectare in both years and their combined data. The genotype HRD CUM 006 x 003 produced significantly the highest total yield
(81.05 mt/ha) followed by HRD CUM 009 x 003 (79.20 $\mathrm{mt} / \mathrm{ha})$ and the lowest yield (17.90 $\mathrm{mt} / \mathrm{ha})$ on Indian hybrid Dynasty inthe first year. In the second year, the genotype HRD CUM 006 x 008 produced significantly the highest total yield $(136.01 \mathrm{mt} / \mathrm{ha})$ followed by HRD CUM 009 x 003 (134.9 mt/ha) and HRD CUM 009 x $007(123.70 \mathrm{mt} / \mathrm{ha})$ whereas the lowest yield $(15.80 \mathrm{t} /$ ha) on HRD CUM 001. Based on the two-year data the genotype HRD CUM 009 x 003 (107.05mt/ha), HRD CUM 006 x 008 (102.25mt/ha) and HRD CUM 004 x $003(99.93 \mathrm{mt} / \mathrm{ha})$ were ranked as first, second and third position, whereas the Indian hybrid Dynasty ranked as last position $(26.10 \mathrm{mt} / \mathrm{ha})$ followed by HRD CUM 001(30.60 mt/ha). Rests of the genotypes produced more than $50 \mathrm{mt} /$ ha yield. The detail yield data in each year and their combined analysis is presented in table 5 .

Table 5. Average yield per plat and adjusted yield per hectare of cucumber under the plastic house at NHRC,Khumaltar during 2018 and 2019

\begin{tabular}{|c|c|c|c|c|c|c|}
\hline \multirow{2}{*}{$\begin{array}{c}\text { Genotypes } \\
\text { Years }\end{array}$} & \multicolumn{3}{|c|}{ Yield per plant } & \multicolumn{3}{|c|}{ Yield (t/ha) } \\
\hline & 2018 & 2019 & Pooled & 2018 & 2019 & Pooled \\
\hline HRD CUM 001 & $5.67 a b c$ & $1.98 a$ & $3.83 a b$ & $45.40 a b c$ & $15.80 a$ & $30.60 a b$ \\
\hline HRD CUM 004 x 001 & $7.06 b c$ & 14.97 defg & 11.02 cde & $56.50 b c$ & $119.70 \mathrm{defg}$ & 88.10 cde \\
\hline HRD CUM 009 x 003 & $9.90 b c$ & $16.86 \mathrm{~g}$ & $13.38 e$ & $79.20 b c$ & $134.90 a$ & $107.05 e$ \\
\hline HRD CUM 009 x 007 & $5.30 a b$ & $15.46 \mathrm{efg}$ & $10.38 c d e$ & $43.00 a b$ & $123.70 \mathrm{efg}$ & 83.35 cde \\
\hline HRD CUM 006 x 003 & $10.90 c$ & $12.70 \mathrm{cdefg}$ & 11.80 cde & $81.50 c$ & 101.60 cdefg & 91.55 cde \\
\hline Dynasty & $2.24 a$ & $4.27 a b$ & $3.26 a$ & $17.90 a$ & $34.29 a b$ & $26.10 a$ \\
\hline HRD CUM 009 x 001 & $7.70 b c$ & 8.97 bcde & 8.34 abcde & $62.30 b c$ & 71.70 bcde & 67.00 abcde \\
\hline HRD CUM 009 x 008 & $8.89 b c$ & 11.49 cdefg & 10.19 cde & $71.10 b c$ & 91.90 edefg & 81.50 cde \\
\hline HRD CUM 006 x 001 & $8.35 b c$ & $4.27 a b$ & $6.31 a b c$ & $66.80 b c$ & $34.20 a b$ & $50.50 a b c$ \\
\hline HRD CUM 006 x 007 & $9.51 b c$ & $8.51 \mathrm{bcd}$ & $9.01 b c d$ & $76.10 b c$ & $68.10 \mathrm{bcd}$ & 72.10 bcde \\
\hline HRD CUM 006 x 008 & $8.54 b c$ & $17.01 \mathrm{~g}$ & $12.78 \mathrm{de}$ & $68.40 b c$ & $136.10 \mathrm{~g}$ & $102.25 \mathrm{de}$ \\
\hline HRD CUM 004 x 003 & $9.15 b c$ & $15.84 f g$ & $12.50 \mathrm{de}$ & $73.16 b c$ & $126.70 \mathrm{fg}$ & 99.93 de \\
\hline HRD CUM 004 x 007 & $7.03 b c$ & $6.77 a b c$ & $6.90 a b c$ & $56.30 b c$ & $54.10 a b c$ & $55.20 a b c$ \\
\hline HRD CUM 004 x 008 & $6.87 b c$ & $11.99 \mathrm{cdef}$ & 9.43 cde & $54.90 b c$ & 95.90 cdefg & 75.40 cde \\
\hline HRD CUM 007 & $6.20 a b$ & $10.73 b c d e f g$ & 8.47 abcde & $43.20 a b$ & 85.80 bcdefg & $64.50 \mathrm{abcd}$ \\
\hline HRD CUM 008 & $5.78 a b c$ & $9.22 \mathrm{bcdfg}$ & $7.50 \mathrm{abcd}$ & $46.30 a b c$ & 73.70 bcdef & $60.00 \mathrm{abcd}$ \\
\hline F-value & $*$ & $* * *$ & $* * *$ & $*$ & $* * *$ & $* * *$ \\
\hline LSD & 3.887 & 5.981 & 4.617 & 31.10 & 47.14 & 4.617 \\
\hline $\mathrm{CV} \%$ & 31.7 & 33.0 & 44.5 & 31.7 & 33.1 & 44.5 \\
\hline
\end{tabular}




\section{Quality parameters}

The observation was also recorded on quality parameters like the colour of the fruit, size, appearance, taste hollowness and hedonic rating by a panel of scientists by visual observation and organoleptic taste during 2017/18. The OP genotype HRD CUM001 (Bhaktpur local) showed significantly the best colour and appearance while the genotype Dhankuta had significantly good marketable size and taste. The absence of hollowness was observed the maximum (2.88 scales) on hybrid genotype HRD CUM 009 x 003 followed by Dhankuta Local (2.77 scales). The highest hedonic rating (8.11 scale) was observed in genotype Dhankuta local. The detail quality parameters in different parameters with their value are presented in table 6.

Table 6.Number of first female flowering and male flowering node of cucumber under the plastic house at NHRC, Khumaltar during 2018

\begin{tabular}{|c|c|c|c|c|c|c|}
\hline Genotypes & $\begin{array}{c}\text { Color* } \\
\text { (1-3 scale) }\end{array}$ & $\begin{array}{c}\text { Size* }^{*} \\
\text { (1-3 scale) }\end{array}$ & $\begin{array}{c}\text { Appearance* } \\
\text { (1-3 scale) }\end{array}$ & $\begin{array}{c}\text { Taste* } \\
\text { (1-3 scale) }\end{array}$ & $\begin{array}{c}\text { Hollowness } \\
\text { (1-3 scale) }\end{array}$ & $\begin{array}{l}\text { Hed. rating } \\
\text { (1-9 scale) }\end{array}$ \\
\hline HRD CUM 001 & $2.88 \mathrm{e}$ & 2.66efg & $2.77 f$ & $2.44 \mathrm{~cd}$ & $2.66 \mathrm{bc}$ & $7.88 \mathrm{ef}$ \\
\hline Dhankuta Local & 2.66de & $2.88 \mathrm{~g}$ & $2.44 \mathrm{cdef}$ & $2.66 \mathrm{~d}$ & $2.77 b c$ & $8.11 \mathrm{f}$ \\
\hline HRD CUM 004 x 001 & $2.33 \mathrm{bcde}$ & $2.22 \mathrm{cdef}$ & 2.11abcde & $2.44 \mathrm{~cd}$ & $2.88 \mathrm{c}$ & $6.77 \mathrm{bcde}$ \\
\hline HRD CUM $009 \times 003$ & $2.00 \mathrm{ab}$ & $2.44 \mathrm{defg}$ & $1.77 \mathrm{ab}$ & $2.22 \mathrm{bcd}$ & $2.66 b c$ & $6.77 \mathrm{bcde}$ \\
\hline HRD CUM 009 x 007 & $2.66 \mathrm{de}$ & $2.55 \mathrm{efg}$ & $2.22 \mathrm{abcdef}$ & $1.55 \mathrm{a}$ & $2.11 a$ & $5.33 \mathrm{a}$ \\
\hline HRD CUM 006 x 003 & $1.66 \mathrm{a}$ & $1.44 \mathrm{a}$ & $1.66 \mathrm{a}$ & $1.66 \mathrm{ab}$ & $2.33 \mathrm{ab}$ & $5.22 \mathrm{a}$ \\
\hline Dynasty & $2.00 \mathrm{ab}$ & $1.88 \mathrm{abcd}$ & $1.88 \mathrm{abc}$ & $2.55 \mathrm{~d}$ & $2.55 \mathrm{abc}$ & $6.88 \mathrm{cde}$ \\
\hline HRD CUM 009 x 001 & 2.11abcd & $1.77 \mathrm{abc}$ & $2.00 \mathrm{abcd}$ & $1.66 \mathrm{ab}$ & $2.33 \mathrm{ab}$ & $5.66 \mathrm{ab}$ \\
\hline HRD CUM 009 x 008 & $2.33 \mathrm{bcde}$ & $2.22 \mathrm{cdef}$ & $2.33 \mathrm{bcdef}$ & $2.66 \mathrm{~d}$ & $2.66 \mathrm{bc}$ & $6.88 \mathrm{cde}$ \\
\hline HRD CUM 006 x 001 & $2.00 \mathrm{ab}$ & $2.22 \mathrm{cdef}$ & $2.00 \mathrm{abcd}$ & $2.11 \mathrm{abcd}$ & $2.55 \mathrm{abc}$ & $6.22 \mathrm{abcd}$ \\
\hline HRD CUM 006 x 007 & $1.66 \mathrm{a}$ & $1.55 \mathrm{ab}$ & $2.11 \mathrm{abcde}$ & $1.66 \mathrm{ab}$ & $2.11 \mathrm{a}$ & $5.33 \mathrm{a}$ \\
\hline HRD CUM 006 x 008 & $2.55 \mathrm{bcde}$ & $3.00 \mathrm{~g}$ & $2.66 \mathrm{ef}$ & $2.66 \mathrm{~d}$ & $2.66 \mathrm{bc}$ & $7.77 \mathrm{f}$ \\
\hline HRD CUM 004 x 003 & $2.55 \mathrm{bcde}$ & $2.77 \mathrm{fg}$ & $2.55 \mathrm{def}$ & $2.33 \mathrm{~cd}$ & $2.77 \mathrm{bc}$ & $7.22 \mathrm{def}$ \\
\hline HRD CUM 004 x 007 & $2.22 \mathrm{abcd}$ & $2.66 \mathrm{efg}$ & $2.44 \mathrm{cdef}$ & $2.22 \mathrm{bcd}$ & $2.55 \mathrm{abc}$ & $6.77 \mathrm{bcde}$ \\
\hline HRD CUM 004 x 008 & $2.00 \mathrm{ab}$ & $2.11 \mathrm{bcde}$ & $1.88 \mathrm{abc}$ & $1.88 \mathrm{abc}$ & $2.66 \mathrm{~b}$ & $5.66 \mathrm{ab}$ \\
\hline HRD CUM 007 & $2.00 \mathrm{ab}$ & $1.88 \mathrm{abcd}$ & $2.11 \mathrm{abcde}$ & $2.44 \mathrm{~cd}$ & $2.33 \mathrm{bc}$ & $6.00 \mathrm{abc}$ \\
\hline Mean & 2.24 & 2.29 & 2.20 & 2.21 & 2.54 & 6.56 \\
\hline F-test & $* * *$ & $* * *$ & $* * *$ & $* * *$ & $* *$ & $* * *$ \\
\hline LSD & 0.490 & 0.531 & 0.448 & 0.515 & 0.446 & 1.037 \\
\hline $\mathrm{CV}$ & 23.4 & 24.8 & 23.8 & 24.9 & 18.8 & 17.0 \\
\hline
\end{tabular}

*means 1-3 scales, where $1=$ the lowest performance value and 3 is the highest performance value.

** 1-3 means hollowness, where $1=$ highest hollow and 3 is the lowest hollow

*** 1-9 scale, where, $1=$ lowest and 9 is the highest performance value.

\section{Coordinated Varietal Trial of Cucumber at DoAR, Tarahara}

The coordinated varietal trial results showed the significant differences on days to first female flowering node, branch number per plant and plant height but not significantly different on days to $50 \%$ female and male flowering among the genotypes (Table 7).The tested genotypes differed significantly on the number of fruits per plant, marketable and total fruit yield /ha but not significantly differed on length, diameter of fruits and un-marketable fruit yield/ha. The hybrid HRD CUC $009 \times 003$ produced the highest total yield (43.54 t/ha) 
and it was statistically at par with HRD CUC 009 x 007 (42.32 mt/ha),Dynasty F1 (41.07),HRD CUC 006 x 003 (DL) (40.23 mt/ha) and HRD CUC 004 x 001 (39.28 $\mathrm{mt} / \mathrm{ha})$ whereas the lowest total yield (25.20 t/ha) was recorded in genotype HRD-CUC 003 (Dhankuta Local) followed by HRD CUC 001 (Bhaktpur Local) (26.0 mt/ ha). Farmers preferred new hybrid HRD CUC 009 x CUC 003 due to high yield and good taste than other tested genotypes. The detail recorded parameters are presented in table8.
The insect and disease scoring showed the nosignificant and significant results respectively (Table 9). The genotypes showed a significant difference in disease scoring (Fusarium wilt). The minimum scoring (1.56 scale) ofFusarium wilt was recorded on the cucumber hybrid dynasty and it was statistically at par with HRD-CUC006 x 003 (1.72 scales) and HRD- CUC009 x 007 (1.78 scales) and the maximum (2.72 scales) on cultivated open-pollinated genotypes HRD-CUCOO1 (Bhaktpur Local) and HRD CUCOO3 (Dhankuta Local).

Table 7. Days to 50 percent male and female flowering, first female node and branch numberof cucumber CVT at DoAR, Tarahara during 2018

\begin{tabular}{|l|c|c|c|c|c|c|}
\hline \multicolumn{1}{|c|}{ Genotypes } & FFFN & DFFF & DFMF & BN & DFH & P Ht. \\
\hline HRD-CUCOO1 (HBL) & $10 \mathrm{abc}$ & 33 & 29 & $4 \mathrm{~b}$ & 34 & $220.7 a$ \\
\hline HRD CUCOO3 (DL) & $11 \mathrm{ab}$ & 34 & 30 & $4 \mathrm{~b}$ & 34 & $230.3 a b$ \\
\hline HRD CUCC004 x 001 & $10 \mathrm{abc}$ & 32 & 26 & $4 \mathrm{~b}$ & 34 & $244.3 \mathrm{bc}$ \\
\hline HRD-CUC009 x 003 & $12 \mathrm{a}$ & 32 & 29 & $4 \mathrm{~b}$ & 34 & $231.0 c d$ \\
\hline HRD- CUC009 x 007 & $9 \mathrm{bc}$ & 32 & 29 & $5 \mathrm{a}$ & 34 & $282.3 d$ \\
\hline HRD-CUC006 x 003 & $8 \mathrm{c}$ & 32 & 28 & $5 \mathrm{a}$ & 34 & $305.3 d$ \\
\hline Dynasty F1 & $8 \mathrm{c}$ & 32 & 28 & $5 \mathrm{a}$ & 34 & $267.0 d$ \\
\hline F-test & $*$ & $\mathrm{~ns}$ & $\mathrm{~ns}$ & $*$ & & $* * *$ \\
\hline LSD (0.05) & 2.060 & - & - & 0.993 & & 31.23 \\
\hline CV (\%) & 11.9 & 4.7 & 0.3 & 12.0 & & 6.9 \\
\hline
\end{tabular}

$\mathrm{DFFF}=$ days to $50 \%$ female flowering, DFMF =days to $50 \%$ male flowering, $\mathrm{FFFN}=$ first female flowering node, $\mathrm{BN}=$ branch no. /plant, DFH=days to first harvesting and $\mathrm{P}$ HT.= plant height $(\mathrm{cm})$ ns $=$ non-significant, $*=$ Significant at $5 \%, * * * *=$ significant at $<1 \%$ levels. Same letter indicate no significant differences.

Table 8. Days to first harvesting, plant height and length and diameter of fruits of cucumber CVT at DoAR, Tarahara during 2018

\begin{tabular}{|l|l|l|l|l|l|l|}
\hline \multicolumn{1}{|c|}{ Genotypes } & \multicolumn{1}{c|}{ LF } & \multicolumn{1}{c|}{ DF } & \multicolumn{1}{c|}{ NoF } & \multicolumn{1}{c|}{ MY } & \multicolumn{1}{c|}{ UMY } & \multicolumn{1}{c|}{ TY } \\
\hline HRD-CUC 001 (HBL) & 18.44 & 35.2 & $9.33 \mathrm{~cd}$ & $19.84 \mathrm{~b}$ & 6.16 & $26.00 \mathrm{~b}$ \\
\hline HRD CUC 003 (DL) & 20.78 & 33.8 & $9.67 \mathrm{~cd}$ & $18.88 \mathrm{~b}$ & 6.32 & $25.20 \mathrm{~b}$ \\
\hline HRD CUC 004 x 001 & 22.08 & 46.0 & $16.33 \mathrm{a}$ & $31.95 \mathrm{a}$ & 7.33 & $39.28 \mathrm{a}$ \\
\hline HRD-CUC 009 x 003 & 24.07 & 43.6 & $14.00 \mathrm{ab}$ & $36.65 \mathrm{a}$ & 6.89 & $43.54 \mathrm{a}$ \\
\hline HRD- CUC 009 x 007 & 23.18 & 47.9 & $13.67 \mathrm{ab}$ & $35.70 \mathrm{a}$ & 6.62 & $42.32 \mathrm{a}$ \\
\hline HRD-CUC006 x 003 & 21.90 & 45.9 & $11.67 \mathrm{bcd}$ & $34.85 \mathrm{a}$ & 5.39 & $40.23 \mathrm{a}$ \\
\hline Dynasty F1 & 24.17 & 43.4 & $13.33 \mathrm{bcd}$ & $35.77 \mathrm{a}$ & 5.30 & $41.07 \mathrm{a}$ \\
\hline F-test & $\mathrm{ns}$ & $\mathrm{ns}$ & $*$ & $* * *$ & $\mathrm{~ns}$ & $* * *$ \\
\hline LSD (0.05) & - & - & 3.673 & 4.734 & - & 6.178 \\
\hline CV (\%) & 11.0 & 18.1 & 16.4 & 8.7 & 39.7 & 9.4 \\
\hline
\end{tabular}

$\mathrm{LF}=$ length of fruit $(\mathrm{cm}), \mathrm{DF}=$ diameter of fruit $(\mathrm{mm}), \mathrm{NoF}=$ fruit numbers/plant, $\mathrm{MY}=$ marketable yield $(\mathrm{t} / \mathrm{ha})$, $\mathrm{UMY}=$ un-marketable yield ( $\mathrm{t} / \mathrm{ha}), \mathrm{TY}=$ Total yield $(\mathrm{t} / \mathrm{ha})$.

ns $=$ non-significant, $*=$ Significant at $5 \%$, ****= significant at $<1 \%$ levels. 
Same letter indicate no significant differences.

Table 9. Insect and disease scoring of cucumber CVT at DoAR, Tarahara during 2018

\begin{tabular}{|l|c|c|}
\hline \multicolumn{1}{|c|}{ Genotypes } & $\begin{array}{c}\text { Insect Scoring } \\
\text { (Fruit fly; 1-5 scale) }\end{array}$ & $\begin{array}{c}\text { Disease Scoring (Fusariumwilt } \\
\text { (1-5 Scale) }\end{array}$ \\
\hline HRD-CUC 001 (HBL) & 1.17 & $2.72 a$ \\
\hline HRD CUC 003 (DL) & 1.20 & $2.72 a$ \\
\hline HRD CUC 004 x 001 & 1.50 & $2.00 a b$ \\
\hline HRD-CUC 009 x 003 & 1.11 & $1.89 a b$ \\
\hline HRD- CUC 009 x 007 & 1.28 & $1.78 b$ \\
\hline HRD-CUC 006 x 003 & 0.72 & $1.72 b$ \\
\hline Dynasty F1 & 0.89 & $1.56 b$ \\
\hline F-test & $\mathrm{ns}$ & $* * *$ \\
\hline LSD $(0.05)$ & - & 0.2614 \\
\hline CV $(\%)$ & 36.7 & 7.1 \\
\hline
\end{tabular}

ns $=$ non-significant and $* * * *=$ significant at $<1 \%$ levels

same letter indicates no significant differences.

Farmers Field Trial of Cucumber at NCRP, Dhankuta

The results of FFT showed that the new hybrid HRD 009x 003 produced $263.64 \%$ more yield at NCRP Dhankuta, farm and $248.90 \%$ more yield at one farmer's field than farmers local genotype HRD CUC003 (Dhankuta Local). The new hybrid also produced $85.86 \mathrm{mt} / \mathrm{ha}$ and $30.08 \mathrm{t} / \mathrm{ha}$ more fruit yield in the other two farmers' fields whereas, HRD CUC003

did not produce any fruits in two farmers' fields. (Table 10).All participated farmers liked new cucumber hybrids HRD 009x HRD003due to its higher yield and good taste than HRD CUC 003 (Dhankuta Local). All farmers requested a large amount of new hybrid seeds for growing in next season.

Table 10.Performance of new cucumber hybrids at NCRP, Dhankuta and farmers' fields during 2018

\begin{tabular}{|l|l|c|c|c|c|c|}
\hline \multicolumn{1}{|c|}{ Farmers name } & \multicolumn{1}{|c|}{ Treatments } & DFH & LF & DF & VL & TY \\
\hline \multirow{3}{*}{ NCRP, Dhankuta } & HRD 009x 003 & 55 & 28.4 & 6.47 & 5.39 & 78.46 \\
\cline { 2 - 7 } & HRD CUC003 & 55 & 35.75 & 7.91 & 7.84 & 29.76 \\
\hline \multirow{3}{*}{ Ghanashyam Ghimire } & HRD 009x 003 & 55 & 30 & 6.94 & 6.0 & 107.08 \\
\cline { 2 - 8 } & HRD CUC003 & 75 & 34.64 & 7.99 & 7.14 & 43.02 \\
\hline \multirow{3}{*}{ Narmada Poudel } & HRD 009x HRD003 & 63 & 33.67 & 6.90 & 7.68 & 85.86 \\
\cline { 2 - 8 } & HRD CUC003 & 0 & 0 & 0 & 0 & 0.00 \\
\hline \multirow{2}{*}{ Dhan K. Rai } & HRD 009x 003 & 53 & 34.6 & 7.08 & 7.1 & 30.08 \\
\cline { 2 - 8 } & HRD CUC003 & 0 & 0 & 0 & 0 & 0.00 \\
\hline
\end{tabular}

$\mathrm{DFH}=$ days to first harvest, $\mathrm{LF}=$ length of fruit $(\mathrm{cm}), \mathrm{DF}=$ diameter of fruit $(\mathrm{cm}), \mathrm{VL}=$ Vine length $(\mathrm{cm})$ and $\mathrm{TY}=$ Total yield ( $\mathrm{t} / \mathrm{ha})$. 


\section{Discussions}

The fruit yield of cucumber depends on the many genetic and environment factors. The genetic parameters govern for higher yield in cucumbers are mainly depends on days to first flowering and the number of nodesat which the first female flowering initiate. Past experiences and many references indicated that the initiation of male and female flowers even in same variety is mainly depends on climatic factors. In high temperature plant produced more male flowers whereas in low temperature there is production of more female flowers and cause the shortage of male flowers for proper fertilization. Based on the these finding pruning practices is recommended differently in different seasons. In summer topping of main stem and subsequent stems is recommended to promote the growth of secondary and tertiarybranches to get more female flowers while removing of side shoots is recommended during the winter season. Besides the temperature and climatic condition genotypes itself play the important role for initiation of male and female flowers in early nodes. In this study genotypes showed significant differences on male to female flower ratio. Among the tested hybrids and open pollinated lines, the lowest male to female flower ratio (1:02) was recorded in new hybrids HRDCUC-006 x 001 and HRDCUC-004 x 003 whereas, the open pollinated variety Bhaktapur local produced the highest male to female ratio (8.6:1). Which clearly indicated that early harvesting and more yield was recorded in genotypes having the ability to produce early female flowering in lowest nodes.

Previously most of the farmers and consumers gave emphasis for production of large fruits, preserved their fruits with in plant for maturity for pickling and fresh consumptions. But now most consumers preferred small size fruits (350-500 gram), more than $15 \mathrm{~cm}$ long with $5-6 \mathrm{~cm}$ girth. Most of the hybrids in this study produced similar shape and size of fruits within 12-15 days after initiation of first female flowers.

The numbers of fruits per plants depend on the plant height, male to female ratio and first female flowering node and duration of crops. In this study most of the hybrids showed the ability to produce a greater number of fruits per plant as compare to local varieties. The low yield in the Indian hybrid than new hybrids could be due to early and short productive life, small plant height, small fruit size and low number of fruits per plant, while higher yield in new hybrids could be due to earliest female flowering, higher number of fruits per plants. It could be due to genetic factors rather than the environment. It is reported that the yield and yield attributing parameters are governed by genetic factor with high heritability. Gangadhar et.al. (2019) reported that similar findings. He mentioned that there was $99.26 \%$ heritability on days to first female flowering, 98.78\% heritability on the fruit number and $96.37 \%$ heritability on fruit yield/plant. He also reported that there was a positive correlation (0.249) on days to first female flower and fruit weight which was in the line of our finding.

For the adaptation of new variety/ hybrids by farmers and consumers is not only depends on yield but it depends on visual observation and quality parameters. The preference of consumers also depends on agroecological conditions and ethnicity. In our country dark green types cucumbers with mealy and soft taste is morepreferred in the eastern part with majority of Gurung and Tamang community while in central and other parts mealy and soft taste with light green cucumbers are more preferred by all ethnicity groups. Based on these above quality parameters hybrid developed by crossing HRD-CUC $009 \times 003$ is recommended for cultivation in the eastern part and hybrids developed by crossing and HRD-CUC 006 × 008 and HRD-CUC 004 x 001 are recommended for central and other parts.

Since there is no exact recommendation of fertilizers for hybrid cucumber production in Nepal and it may vary according to soil condition. The wide variation of yield in two years could be due to the response of genotypes on fertilizers rate. The addition of $50 \mathrm{~g}$ mustard cake, $50 \mathrm{~g}$ bone meal and top-dressing of $3 \mathrm{~g}$ each micronutrient (containing bio zyme, zinc and boron) in the second year could be the main reason for higher production of cucumber in second years. The low yield of open-pollinated variety HRD CUM 001 (Bhaktpur Local) in the second year was due to heavy infestation of the plant by powdery mildew; possibly to the negative effect of the heavy dose of nutrients. This clearly indicated urgent necessity for conducting a separate fertilizers experiment for the hybrid cucumber production. 


\section{Conclusion}

Based on the results of two years field experiment under the plastic house at NHRC and a one-year open field experiment in DoAR, Tarahara and farmers field trial in Dhankuta, the new cucumber hybrids developed by crossing HRD-CUC $009 \times 003$ is recommended for cultivation in the eastern part of Nepal due to higher yield (43.5 mt/ha)in the open field, preferred green fruit colour and other quality parameters. In the central part, the cucumber hybrids developed by crossing and HRDCUC 006 x $008(102.25 \mathrm{mt} / \mathrm{ha})$ and HRD-CUC 004 x $001(88.10 \mathrm{mt} / \mathrm{ha})$ are promising for cultivation under plastic house due to their higher yield performance, taste and greenish-white colour of fruits. Further one to two years verification trail is suggested in the central and western part of the county before registration/ release of varieties.

\section{Acknowledgement}

The authorswould like to acknowledge Nepal Agriculture Research Council (NARC) for approving project and financial support. We also acknowledge Dr. Madhu Sudhan Ghale and Dr Krishna Prasad Poudel former chiefs for collection of gynoecious lines. Wewould also like to express thanks to all staffs of NHRC, Khumaltar and participating farmers for their support for conducting field experiment.

\section{Declaration of conflict of interest and ethical approval:}

The authors declare that they don't have any competing interests on the published materials.

\section{References}

FAO, STAT. (2021). Food and Agriculture Organization. Retrieved on 16, January2021.

Gangadhar, K., Kumar, R., Selvakumar, R., Apparao, V.V. and Yadav, L.P. (2019). Evaluation of cucumber hybrids/lines for yield and quality under polyhouse. International Journal of Current Microbiology and Applied Sciences. ISSN 2319-7706, Volume 8:6. DOI:https://doi. org/10-20546/ijcmas.2019.806.197

Gautam, I.P., Upadhyay, K. P., Chaudhary, J.N., Khan, A.H. and Timilsina, C.K. (2008). Evaluation of cucumber genotypes for open field and plastic house in the western hills of Nepal. Proceedings of the Third SAS-N Convention, 27-29 August 2008, Kathmandu, Nepal,197-203. ISSN 16826566.

Kafe, A. and Joshi, K.R. (2018). Vegetable Seed Import Scenario in Nepal. www.researchgate. net $>$ publication $>327511348$-Vegeta...Pdf. Retrieved on 22, September 2020.

MOALD. (2018/19). Ministry of Agriculture and Livestock Development. Statistical Information on Nepalese Agriculture. Govern of Nepal, Statistics and Analysis Section, Singh Durbar, Kathmandu, Nepal.

Pal, S., Sharma, H.R. and Yadav, N. (2017). Evaluation of Cucumber Genotypes for Yield and Quality Traits. Journal of Hill Agriculture 8(2):144-150. DOI:https://doi:10.5958/2230-7338.2017.00027

Rongsen, L. 1994. The basic principles of plastic film technology. The application of plastic film technology in China.ICIMOD, Kathmandu, Nepal, pp.11.

Srivastava, B.K. and M.P. Singh. 1997. Boosting offseason production of Vegetables in Low-Cost Polyhouse. Proc. Third Agriculture Science Congress, PAU, Ludhiana, March 12-17. 